\title{
Self-efficacy beliefs and the relation between career planning and perception of barriers
}

\author{
Paulo Cardoso · João Manuel Moreira
}

Received: 16 March 2008/Accepted: 29 October 2008/Published online: 22 September 2009

(C) Springer Science+Business Media B.V. 2009

\begin{abstract}
This study tested the hypothesis that self-efficacy in career roles moderates the relation between perception of career barriers and career planning, in a study with Portuguese students, 488 in Grade 9 and 517 in Grade 12. The results supported the hypothesis only among Grade 9 girls, showing that perception of career barriers leads to less career planning among girls low in self-efficacy. The findings suggest that variables such as gender and psychological maturity need to be considered in understanding career planning.
\end{abstract}

Résumé. Croyances d'auto-efficacité et relation entre la planification de carrière et la perception des barrières. Cette étude évalue l'hypothèse, dans une étude menée sur des étudiants portugais, 488 en $9^{\mathrm{e}}$ année et 517 en 12e, que l'autoefficacité dans les rôles professionnels modère la relation entre la perception des barrières de carrière et la planification de carrière. Les résultats soutiennent l'hypothèse seulement pour les filles de 9e année, prouvant que la perception des barrières de carrière mène à moins de planification de carrière parmi les filles de faible niveau d'auto-efficacité. Les résultats suggèrent que des variables telles que le genre et la maturité psychologique doivent être pris en considération dans la compréhension de la planification de carrière.

Zusammenfassung. Selbsteinschätzungen der eigenen Durchsetzungsfähigkeit und die Beziehung zwischen Berufsplanung und der Wahrnehmung von Hemmnissen. In einer Untersuchung mit portugiesischen Schülern, 488 in Klasse 9 und 517 in Klasse 12, wurde die Hypothese überprüft, dass die Einschätzung der

P. Cardoso $(\bowtie)$

Departamento de Psicologia, Universidade de Évora, Apartado 94, 7000-554 Évora, Portugal

e-mail: pmsc@uevora.pt

J. M. Moreira

Universidade de Lisboa, Lisbon, Portugal 